\title{
Colonial choanoflagellate isolated from Mono Lake harbors a microbiome
}

Hake, K. H. ${ }^{1,2}$, West, P.T. ${ }^{3}$, McDonald, K. ${ }^{4}$, Laundon, D. ${ }^{5}$, Garcia De Las Bayonas, A. ${ }^{\text {, }}$ Feng, C. ${ }^{1}$, Burkhardt, P. ${ }^{5,6}$, Richter, D.J. ${ }^{7}$, Banfield, J.F. ${ }^{3}$, and King, N. ${ }^{1}{ }^{*}$

${ }^{1}$ Howard Hughes Medical Institute and Department of Molecular and Cell Biology, University of California, Berkeley, CA, USA

${ }^{2}$ Present address: Calico Life Sciences, South San Francisco, CA, USA

${ }^{3}$ Department of Environmental Science, Policy, \& Management, University of California, Berkeley, CA, USA

${ }^{4}$ Electron Microscopy Laboratory, University of California, Berkeley, CA, USA

${ }^{5}$ Marine Biological Association of the United Kingdom, Plymouth, United Kingdom

${ }^{6}$ Sars International Centre for Molecular Marine Biology, University of Bergen, Bergen, Norway

20

${ }^{7}$ Institut de Biologia Evolutiva (CSIC-Universitat Pompeu Fabra), Barcelona, Spain

*Send correspondence to nking@berkeley.edu 


\begin{abstract}
Choanoflagellates offer key insights into bacterial influences on the origin and early evolution of animals. Here we report the isolation and characterization of a new colonial choanoflagellate species, Barroeca monosierra, that, unlike previously characterized species, harbors a stable microbiome. B. monosierra was isolated from Mono Lake, California and forms large spherical colonies that are more than an order of magnitude larger than those formed by the closely related Salpingoeca rosetta. By designing fluorescence in situ hybridization probes from metagenomic sequences, we found that $B$. monosierra colonies are colonized by members of the halotolerant and closely related Saccharospirillaceae and Oceanospirillaceae, as well as purple sulfur bacteria (Ectothiorhodospiraceae) and non-sulfur Rhodobacteraceae. This relatively simple microbiome in a close relative of animals presents a new experimental model for investigating the evolution of stable interactions among eukaryotes and bacteria.
\end{abstract}

\title{
IMPORTANCE
}

The animals and bacteria of Mono Lake (California) have evolved diverse strategies for surviving the hypersaline, alkaline, arsenic-rich environment. We sought to investigate whether the closest living relatives of animals, the choanoflagellates, exist among the relatively limited diversity of organisms in Mono Lake. We repeatedly isolated members of a single species of choanoflagellate, which we have named Barroeca monosierra, suggesting that it is a stable and abundant part of the ecosystem. Characterization of $B$. monosierra revealed that it forms large spherical colonies that each contain a microbiome, providing an opportunity to investigate the evolution of stable physical associations between eukaryotes and bacteria. 


\section{DISCOVERY REPORT}

\section{A newly identified choanoflagellate species forms large colonies that contain a microbiome}

Choanoflagellates are the closest living relatives of animals and, as such, provide

60 insights into the origin of key features of animals, including animal multicellularity and cell biology [1,2]. Over a series of four sampling trips to Mono Lake, California (Fig. 1A; Table S1) we collected single-celled choanoflagellates and large spherical choanoflagellate colonies, many of which were hollow (Fig. 1B) and resembled the blastula stage of animal development. In colonies and single cells, each cell had the

65 typical collar complex observed in other choanoflagellates: an apical flagellum surrounded by a collar of microvilli $[1,2]$. In these "rosette" colonies, the cells were oriented with the basal pole of each cell pointing inwards and the apical flagellum facing out (Fig. 1). To study the Mono Lake choanoflagellates in greater detail, we established clonal strains from ten independent isolates, two of which were each started from a

70 single-celled choanoflagellate and the remaining eight of which were each started from a single colony (Table S1). The two strains started from single-celled choanoflagellates, isolates ML1.1 and ML1.2, took on the colonial morphology observed in the other isolates after culturing in the laboratory, suggesting that the colonies and single cells isolated from Mono Lake could belong to the same species. We are aware of no prior

75 reports of choanoflagellates having been cultured from any alkaline soda lake, including Mono Lake.

The 18S rDNA genes for six of the Mono Lake isolates were sequenced and found to be $>99 \%$ identical (Table S1). Phylogenetic analysis confirmed that all of the isolates are members of a single choanoflagellate species (Fig. S1). In further phylogenetic analyses based on 18S rDNA and two protein-coding genes from isolate ML2.1 (Fig. 1C) [3], we found that its closest relatives are the emerging model choanoflagellate S. rosetta [4-8], additional Salpingoeca spp. [9] and Microstomoeca roanoka $[3,10]$. The phylogenetic distance separating the Mono Lake species from its closest relatives is similar to the distance separating other choanoflagellate genera. Therefore, we propose the name Barroeca monosierra, with the genus name inspired by esteemed choanoflagellate researcher Prof. Barry Leadbeater and the species name inspired by the location of Mono Lake in the Sierra Nevada mountain range. (See Supplemental Methods for further details and a formal species description.)

Although $B$. monosierra and $S$. rosetta form rosette-shaped spherical colonies, they differ greatly in size. S. rosetta colonies range from 10-30 $\mu \mathrm{m}$ in diameter while $B$. monosierra forms among the largest choanoflagellate colonies observed [1,11], with a single culture exhibiting colony sizes spanning from 10-120 $\mu \mathrm{m}$ in diameter (Fig. 1D-F).

95 Unlike the rosettes of $S$. rosetta, in which the basal poles of cells are closely apposed in the rosette center $[5,7,11,12]$, cells in large $B$. monosierra rosettes form a shell on the surface of a seemingly hollow sphere. Inside the ostensibly hollow sphere, a branched network of extracellular matrix connects the basal poles of all cells (Fig. S2.) 
Upon staining B. monosierra with the DNA dye Hoechst 33342, we observed the expected toroidal nuclei in each choanoflagellate cell [12,13], but were surprised to detect Hoechst-positive material in the interior of $B$. monosierra colonies (Fig. 2A, $A^{\prime}$ ). Transmission electron microscopy revealed the presence of $1 \mu \mathrm{m}$ and smaller cells with diverse morphologies bounded by cell walls in the centers of rosettes (Fig. 2B, B'; Fig.

105 S3). Together, these observations led us to hypothesize that the centers of $B$. monosierra colonies contain bacteria.

By performing hybridization chain reaction fluorescence in situ hybridization (HCR-FISH [14-16]) with a broad-spectrum probe of bacterial 16S rRNA, EUB338 [17], 110 we confirmed that the cells in the center of colonies are bacteria (Fig. 2C). A second probe that specifically targeted $16 \mathrm{~S}$ rRNA sequences from Gammaproteobacteria, GAM42a, revealed that the majority of the bacteria inside the colonies are Gammaproteobacteria (Fig. 2C')[18]. Finally, by incubating B. monosierra cultures with fluorescently labeled D-amino acids, which are specifically incorporated into the cell

115 walls of growing bacteria, we found that the bacteria in $B$. monosierra colonies are alive and growing (Fig. S4)[19]. Therefore, B. monosierra contains a microbiome as defined in [20].

To visualize the spatial distribution of choanoflagellate and bacterial cells in a 120 representative colony, we generated a 3D reconstruction from serial sections imaged by TEM. The colony contained 70 choanoflagellate cells that were tightly packed, forming a largely continuous monolayer of cells (Fig. 2D). As observed by immunofluorescence microscopy (Figs. 2A and 2A'), all cells were highly polarized and oriented with their apical flagella and collars extending away from the centroid of the rosette. Many cells

125 were connected by fine intercellular bridges (Fig. S5) that have been previously observed in other colonial choanoflagellates, including $S$. rosetta $[5,12]$.

The 3D reconstruction also revealed at least 200 bacterial cells in the center of the rosette (Fig. 2D', 2D"), some of which were physically associated with and wrapped

130 around the choanoflagellate ECM (Fig. S6). A small number of bacterial cells were observed between the lateral surfaces of choanoflagellate cells, although it was not possible to determine whether they were entering or exiting the colony (Fig. 2D"; Fig. S7). Colonies failed to incorporate bacteria-sized bovine serum albumin (BSA)-coated latex microspheres $(0.2 \mu \mathrm{m}$ and $1 \mu \mathrm{m})$ into their centers, suggesting that environmental

135 bacteria may not be capable of passively accessing the centers of $B$. monosierra colonies (Fig. S8).

\section{Gammaproteobacteria and Alphaproteobacteria in the B. monosierra microbiome}

We next sought to identify which bacteria comprise the microbiomes of $B$. monosierra. To identify candidate bacteria for which to design FISH probes, we first sequenced and assembled metagenomes and 16S rDNA sequences from choanoflagellate-enriched samples and from environmental bacteria-enriched samples. These samples were derived from two co-cultures of $B$. monosierra with Mono Lake bacteria, ML2.1E and ML2.1G (Fig. S9), with the enrichment for choanoflagellates or 
bacteria performed by centrifugation. A total of 24 different bacterial species were identified via two complementary bioinformatic approaches (EukRep Metagenomic Analysis and EMIRGE 16S rRNA Analysis; Table S2), of which 22 species were present in fractions enriched with $B$. monosierra colonies (Table S3). The phylogenetic relationships among these and other bacterial species were determined based on analysis of highly conserved ribosomal proteins and 16S rDNA sequences (Fig. 2E and S10).

The 22 bacterial species detected in cultures with $B$. monosierra may have co-

155 sedimented with the $B$. monosierra colonies due their community-structure densities (e.g. biofilms), a transient association with the choanoflagellate colonies (e.g. as prey), or through a stable association with the choanoflagellate colonies. Upon investigation by FISH microscopy, we detected ten or eleven of these species in the centers of $B$. monosierra colonies (Table S4, Fig. S11). (The uncertainty regarding the precise

160 number of choanoflagellate-associated bacterial species stems from the inability to disambiguate $16 \mathrm{~S}$ rDNA sequences corresponding to one or two of the species.) Of these microbiome bacteria, nine were Gammaproteobacteria from the families Oceanospirillaceae (Fig. S11A; OceaML1, OceaML2, OceaML3, OceaML4, OceaML4), Ectothiorhodospiraceae (Fig. S11B; EctoML1, EctoML2, EctoML3, EctoML4), and

165 Saccharospirillaceae (Fig. S11C; SaccML), matching our original observation that the majority of the bacteria were Gammaproteobacteria (Fig. 2C, C'). The remaining species was a Roseinatronobacter sp. (RoseML; Alphaproteobacteria) (Fig. S11D). The microbiome bacteria exhibited an array of morphologies, from long and filamentous to rod shaped (Fig. S3 and Fig. S12). Intriguingly, with the exception of OceaML3, which

170 was exclusively detected inside B. monosierra colonies of ML2.1E (Fig. S13), all other microbiome species identified in this study were detected both inside and outside the colonies.

In animals, the microbiome often contains a core set of host-adapted bacteria 175 that are present in many or all individuals in a host species, as well as a more flexible set of bacteria that may be found in only a subset of individuals [21-23]. To identify core members of the $B$. monosierra microbiome, we measured the frequency with which colonies of ML2.1EC contained or lacked a number of representative microbiome bacteria (Fig. S14A) and estimated the abundance of each species relative to the total 180 microbiome (Fig. S14B). Only one bacterium tested, OceaML1, was found in the microbiome of all B. monosierra colonies (Fig. S14A). The other most frequently observed members of the microbiome were SaccML (93.3\% of colonies), EctoML3 (91.8\% of colonies) and EctoML1 (82.4\% of colonies; Fig. S14A). Two other Gammaproteobacterial species, OceaML2 and EctoML2, were found in $\sim 50-60 \%$ of 185 colonies, while the Alphaproteobacterium RoseML was found in only $13.9 \%$ of rosettes.

The most common resident of the $B$. monosierra microbiome, OceaML1, was also the most abundant, representing on average $66.4 \%$ of the total bacterial load per colony (Fig. S14B). Other abundant bacteria, some found in $>80 \%$ of colonies, represented 190 approximately smaller percentages of the average bacterial biomass in the microbiomes in which they were found. For example, SaccML was found in $93.3 \%$ of 
microbiomes but represented only $30.3 \%$ of total bacteria in the $B$. monosierra rosettes in which it was found. EctoML1, which was found in $82.4 \%$ of $B$. monosierra rosettes represented less than $10 \%$ of the bacteria in the bacteria in which was detected. Thus, only OceaML1 appears to be a core member of the B. monosierra microbiome. Other symbionts detected in B. monosierra (e.g. SaccML and OceaML2-4) are close relatives of OceaML1 and may engage in similar metabolic or developmental functions in their interactions with $B$. monosierra [24].

\section{Discussion}

Interactions with bacteria are essential to choanoflagellate nutrition and life history. Bacteria are the primary food source for choanoflagellates, and the

205 choanoflagellate $S$. rosetta responds to different secreted bacterial cues to undergo either multicellular developmental or mating [25-28]. We report the isolation and characterization of a new choanoflagellate species, B. monosierra, that forms large colonies and contains a microbiome consisting of at least ten different bacterial symbionts. To our knowledge, this is the first example of a stable interaction between

210 choanoflagellates and ectosymbiotic bacteria. Future studies will be important to determine how the $B$. monosierra colonies and their bacterial symbionts interact.

We detected $10-11$ bacterial species from four different families (Saccharospirillaceae, Oceanospirillaceae, Ectothiorhodospiraceae, and

215 Rhodobacteraceae) in the B. monosierra microbiome. Comparisons with other symbioses suggest that their interactions with $B$. monosierra may relate to metabolism and detoxification of environmental sulfur. Such functions have been reported for Oceanospirillaceae species that are symbionts of diverse marine animals, from corals to snails [29-36] and for Ectothiorhodospiraceae that are symbionts of diverse ciliates and 220 animals [37-40].

Although the composition of the animal gut microbiome often varies between individuals in a species, many host species harbor a core set of microbes, with which they participate in stable metabolic interactions and may coevolve [22,23]. Indeed,

225 choanoflagellates express homologs of Toll-like receptors that, in animals, mediate interactions with gut bacteria to maintain homeostasis [10,41]. In $B$. monosierra, OceaML1 was found in $100 \%$ of rosettes assessed and was the most abundant bacterium in the microbiome, suggesting that its interactions with $B$. monosierra may be essential for $B$. monosierra biology. Interestingly, the related bacterium

230 Endozoicomonas sp. (Order Oceanospirillales), is a core member of the gut microbiome of the ascidian Ciona intestinalis, where it represents up to $54 \%$ of the bacterial biomass [32].

B. monosierra and its associated microbiome provide a unique opportunity to 235 characterize a new symbiotic interaction between a single choanoflagellate species and a microbial community. Due to the phylogenetic relevance of choanoflagellates, this symbiotic relationship has the potential to illuminate the ancestry of and mechanisms 
bioRxiv preprint doi: https://doi.org/10.1101/2021.03.30.437421; this version posted June 14, 2021. The copyright holder for this preprint (which was not certified by peer review) is the author/funder, who has granted bioRxiv a license to display the preprint in perpetuity. It is made available under aCC-BY 4.0 International license.

underlying stable associations between animals and bacteria, colonization of organisms by diverse microbial communities, and insights into one of the most complex animalbacterial interactions, the animal gut microbiome. 
245 listed in Table S6. Sequences for B. monosierra 18S rDNA, EFL, and Hsp90 (Fig. 1C) have been assigned GenBank accession numbers MW838180, MW979373 and MW979374, respectively. 18S sequences for different $B$. monosierra strains (Fig. S1, Table S1) have been assigned GenBank accession numbers MZ015010-MZ015015. The assembled $B$. monosierra genome sequence is currently being uploaded to

250 GenBank and will be available under the accession number PRJNA734368. The $B$. monosierra genome, all bacterial genome sequences, and all relevant input and output data from the phylogenetic trees presented in Fig. 1C and Fig. S1 are available via FigShare (https://doi.org/10.6084/m9.figshare.14474214).

255 Author contributions: P.W. performed metagenomic analysis, K.M. did the transmission electron microscopy, D.L. and P.B. did the TEM-reconstruction, and C.F. helped culture B. monosierra. A.G.D.L.B. imaged the theca. K.H. performed all other experiments and analysis. D.J.R. originally isolated B. monosierra and contributed to manuscript editing and the taxonomic description. J.B. and N.K. contributed to project 260 leadership, experimental design, figure design, writing, and editing.

\section{Acknowledgements}

We thank Tarja Hoffmeyer for help with establishing some of the original B. monosierra 265 cultures. We thank Emil Ruff and Julia Schwartzman for assistance with early CARDFISH experiments through the Physiology Course and Microbial Diversity Course at the Marine Biological Laboratory in Woods Hole. We thank Michael VanNieuwenhze for the fluorescent D-amino acids. Frank Nitsche provided advice on the formulation of an artificial Mono Lake culture medium. We thank the following for helpful discussions and

270 research support: Reef Aldayafleh, Cédric Berney, David Booth, Ben Larson, Monika Sigg, Laura Wetzel, and Arielle Woznica. We thank Karen Carniol for valuable feedback on the manuscript. This material is based upon work supported by the National Science Foundation Graduate Research Fellowship under Grant No. DGE 1106400 and DGE 1752814. D.J.R. received the support of a fellowship from "la Caixa" Foundation (ID $275100010434)$ with the fellowship code LCF/BQ/PI19/11690008. 


\section{Supplement Contents}

280 Text S1: Supplemental Materials and Methods

Figure S1: Ultrastructure and phylogeny of choanoflagellate isolates from Mono Lake Figure S2. B. monosierra colonies contain an extracellular matrix (ECM).

Figure S3: Bacterial symbionts in $B$. monosierra colonies exhibit a range of 285 morphologies.

Figure S4: Bacteria inside $B$. monosierra colonies are alive and growing.

Figure S5: Intercellular bridges connect cells in B. monosierra colonies.

Figure S6: Bacterial symbionts physically associate with and wrap around the choanoflagellate ECM.

290 Figure S7: Bacteria are found wedged between the lateral surfaces of $B$. monosierra cells.

Figure S8: $B$. monosierra colonies cannot be passively penetrated by sub-micron particles.

Figure S9: Schematic describing the establishment of $B$. monosierra cultures.

295 Figure S10: Phylogenetic analysis of 16S rRNA tree of EMIRGE sequences.

Figure S11: Diverse bacteria are found in the microbiome of $B$. monosierra.

Figure S12: $B$. monosierra symbionts exhibit filamentous and rod morphologies.

Figure S13: The symbiont OceaML3 is present exclusively inside rosettes.

Figure S14: OceaML1 is a core member of the B. monosierra microbiome.

Table S1: Date, location, phenotype, and isolate designations for Barroeca monosierra isolates.

Table S2: Nine genera of bacteria identified in $B$. monosierra cultures through two independent analyses: comparison of ribosomal proteins detected through

305 metagenomic assembly and by 16 s rRNA assembly and analysis.

Table S3: Predicted targets of HCR-FISH probes based on $16 \mathrm{~S}$ rRNA sequences. Bacteria in bold were identified inside $B$. monosierra colonies.

Table S4: Full length probes, with spacer and initiator sequences, used for HCR-FISH. Table S5: Growth Media Recipes.

310 Table S6: Bacterial 16S rDNA - Genbank accession numbers. 
bioRxiv preprint doi: https://doi.org/10.1101/2021.03.30.437421; this version posted June 14, 2021. The copyright holder for this preprint (which was not certified by peer review) is the author/funder, who has granted bioRxiv a license to display the preprint in perpetuity. It is made available under aCC-BY 4.0 International license.

A

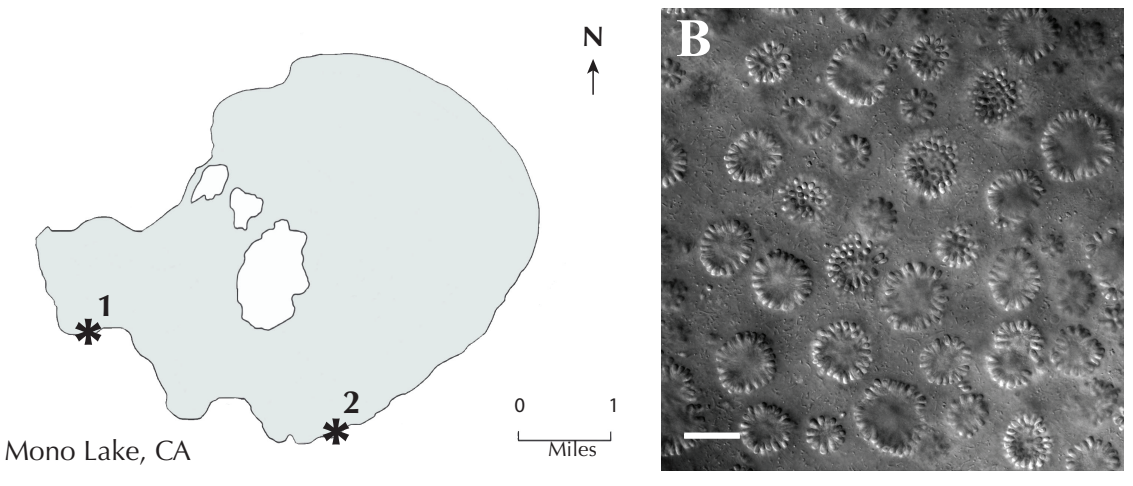

C
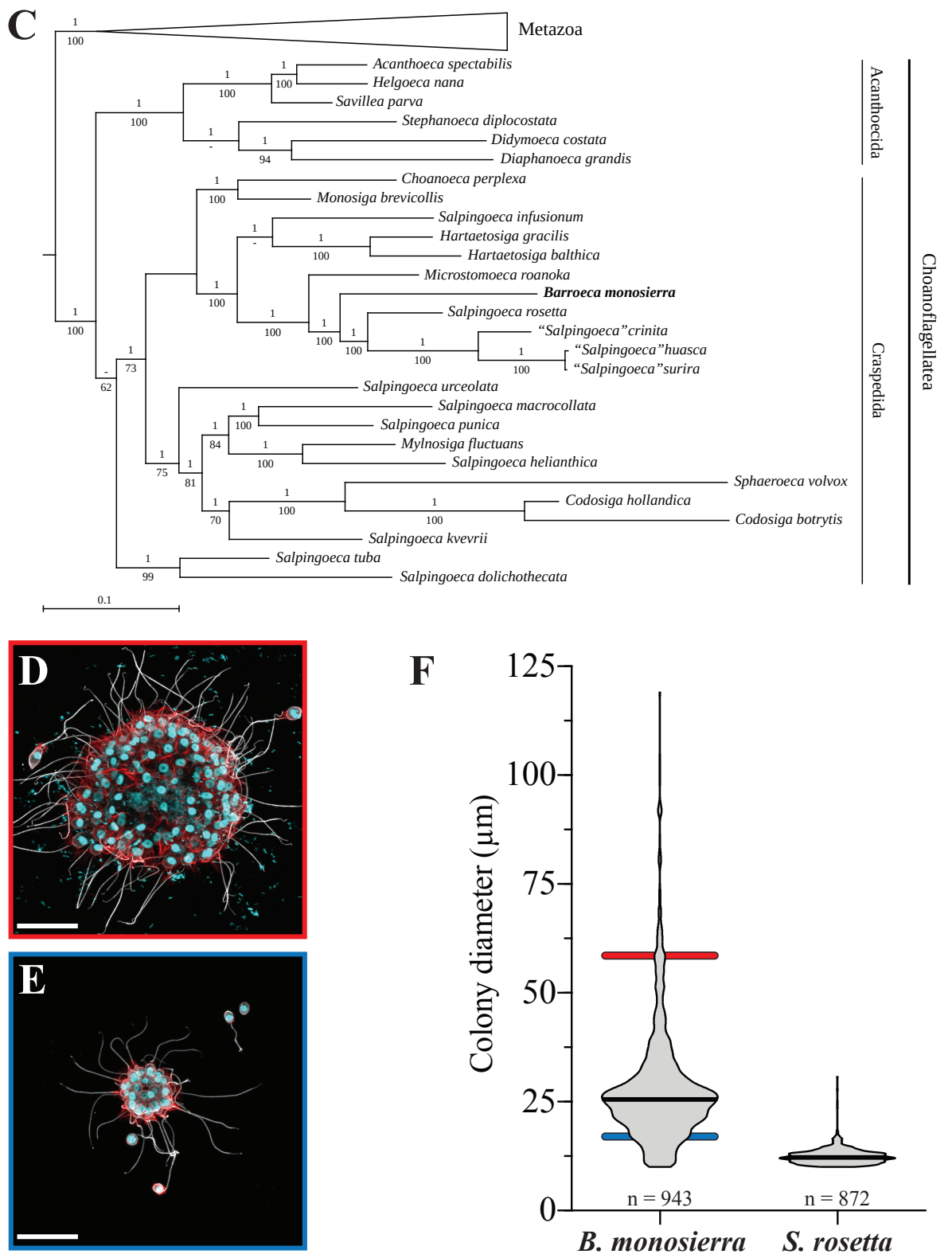
Figure 1. A new colony-forming choanoflagellate isolated from Mono Lake.

(A) Choanoflagellates were collected from two sampling sites (asterisks) near the shore of Mono Lake, California. (Modified from map at monolake.org.) (B) B. monosierra forms large colonies (DIC image). Scale bar $=50 \mu \mathrm{m}$. (C) B. monosierra (shown in bold) is a craspedid choanoflagellate closely related to $S$. rosetta and Microstomoeca roanoka. Phylogeny based on sequences of 3 genes: 18S rDNA, EFL and HSP90. Metazoa (7 species) were collapsed to save space. Bayesian posterior probabilities are indicated above each internal branch, and maximum likelihood bootstrap values below. (A '-' value indicates a bifurcation lacking support or not present in one of the two

325 reconstructions.) (D-E) Two representative colonies reveal the extremes of the $B$. monosierra colony size range $(\mathrm{D}, 58 \mu \mathrm{m}$ diameter; $\mathrm{E}, 19 \mu \mathrm{m}$ diameter; scale bar $=20$ $\mu \mathrm{m})$. In B. monosierra colonies, each cell is oriented with its apical flagellum (white; labeled with anti-tubulin antibody) and the apical collar of microvilli (red; stained with phalloidin) pointing out. Nuclei (cyan) were visualized with the DNA-stain Hoechst

330 33342. (F) Colonies of B. monosierra span from $10 \mu \mathrm{m}$ in diameter, a size comparable to that of small $S$. rosetta colonies, to $120 \mu \mathrm{m}$, over an order of magnitude larger.

Diameters of $B$. monosierra and $S$. rosetta colonies were plotted as a violin plot; median indicated as thick black line. Diameters of representative colonies indicated as colored bars behind violin plot ( $D$, red bar; $E$, blue bar). 
bioRxiv preprint doi: https://doi.org/10.1101/2021.03.30.437421; this version posted June 14, 2021. The copyright holder for this preprint (which was not certified by peer review) is the author/funder, who has granted bioRxiv a license to display the preprint in perpetuity. It is made available under aCC-BY 4.0 International license.
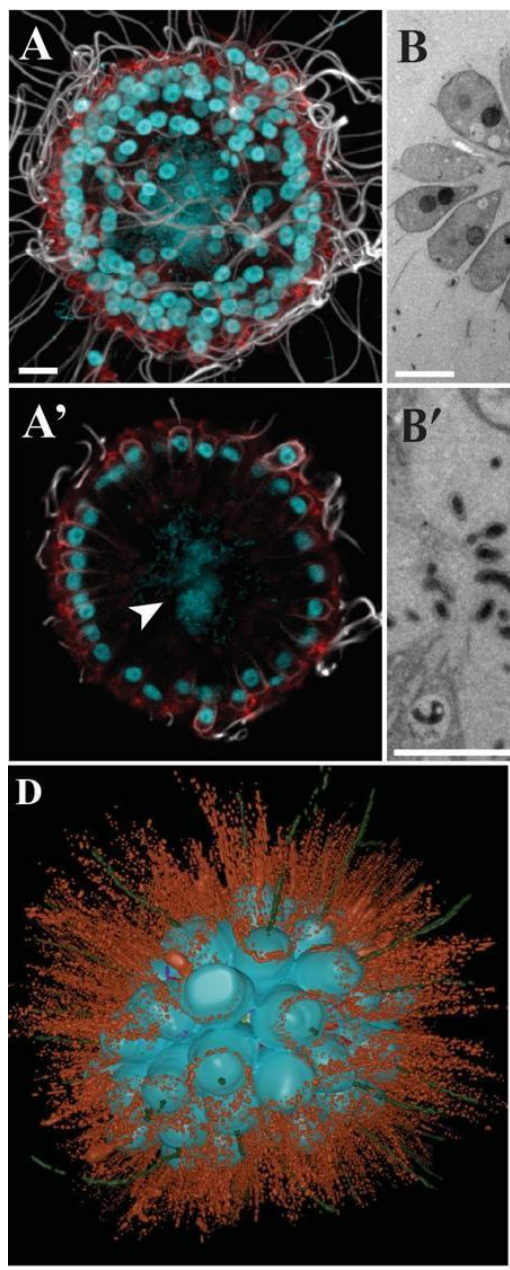
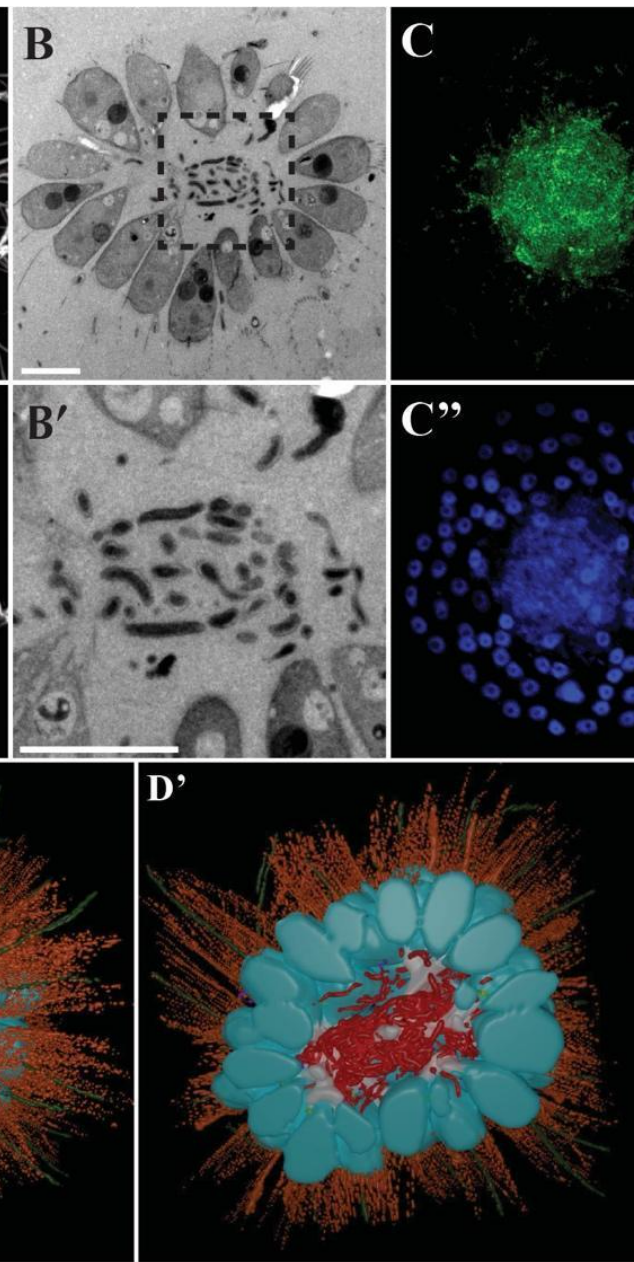
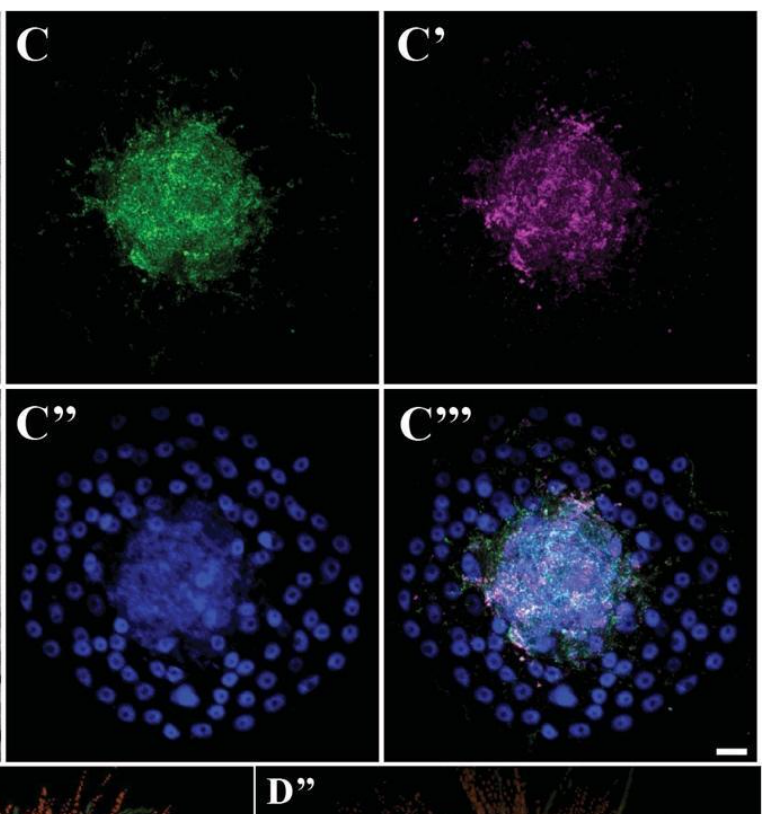

$\mathbf{E}$

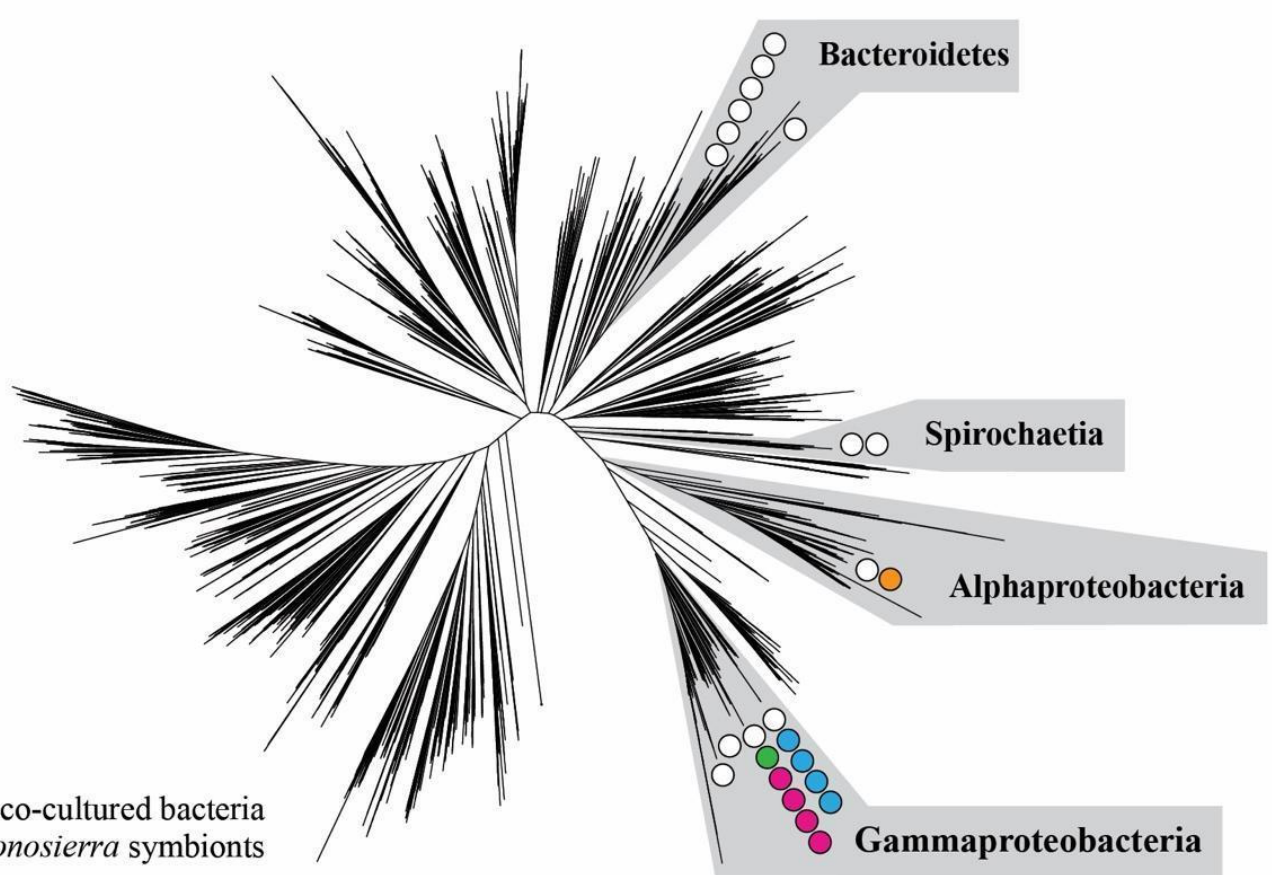

Free-living co-cultured bacteria ○००० $\mathrm{S}$. monosierra symbionts 
Figure 2. B. monosierra colonies are filled with bacteria.

$\left(A, A^{\prime}\right)$ The center of a representative $B$. monosierra colony, shown as a maximum

345 intensity projection $(A)$ and optical z-section ( $\left.A^{\prime}\right)$, contains DNA (revealed by Hoechst 33342 staining; cyan). Apical flagella were labeled with anti-tubulin antibody (white); microvilli were stained with phalloidin (red). Hoechst 33342 staining (cyan) revealed the toroidal choanoflagellate nuclei along the colony perimeter and an amorphous cloud of DNA sitting within the central cavity formed by the monolayer of choanoflagellate cells.

350 (B-B') Thin section through a representative $B$. monosierra colony, imaged by transmission electron microscopy (TEM), revealed the presence of small cells in the central cavity. (B') Inset (box; panel B') reveals that the interior cells are each surrounded by a cell wall. (C-C'”) The small cells inside B. monosierra colonies are bacteria, as revealed by hybridization with a broad spectrum 16S rRNA probe (C,

355 green) and a probe targeting Gammaproteobacteria (C', red). Choanoflagellate nuclei and bacterial nucleoids were revealed by staining with Hoechst (C', cyan). (C"') Merge of panels $C-C$ ". Scale bar for all $=5 \mu \mathrm{m}$. (D-D") 3D reconstruction of a 70-cell $B$. monosierra choanoflagellate colony from transmission electron micrographs of serial ultrathin sections revealed that the bacteria are closely associated with and wrapped 360 around the ECM inside the colony. (D) Whole colony view. (D') Cut-away view of colony center. Color code: cell bodies (cyan); microvilli (orange); flagella (green); bacteria (red); ECM (white); intercellular bridges (yellow, see also Fig. S5); filopodia (purple). (D") Reducing the opacity of the choanoflagellate cell renderings revealed the presence of bacteria positioned between the lateral surfaces of choanoflagellate cells (brackets, see

365 also Fig. S7). (E) Unrooted phylogenetic tree based on 16 concatenated ribosomal protein sequences representing bacterial diversity modified from [42], illustrated to indicate the phylogenetic placement of bacteria co-cultured from Mono Lake with $B$. monosierra. The bacteria belonged to four major classes: Spirochaetia, Alphaproteobacteria, Gammaproteobacteria, and Bacteroidetes, however the bacteria 370 found associated with B. monosierra colonies came only from Alphaproteobacteria and Gammaproteobacteria. Circles represent the phylogenetic placement of non-symbionts (white) and symbionts (Oceanospirillaceae sp., magenta; Saccharospirillaceae sp., green; Ectothiorhodospiraceae sp., blue; Roseinatronobacter sp., orange). See also Figs. S10 and S11. 


\section{References}

1. Leadbeater BSC. The Choanoflagellates: Evolution, Ecology, and Biology. Cambridge, UK: Cambridge University Press; 2015. doi:10.1017/cbo9781139051125

2. Brunet T, King N. The Origin of Animal Multicellularity and Cell Differentiation. Developmental Cell. Cell Press; 2017. pp. 124-140. doi:10.1016/j.devcel.2017.09.016

3. Carr M, Richter DJ, Fozouni P, Smith TJ, Jeuck A, Leadbeater BSC, et al. A six-gene phylogeny provides new insights into choanoflagellate evolution. Mol Phylogenet Evol. 2017;107: 166-178. doi:10.1016/j.ympev.2016.10.011

4. Booth DS, Szmidt-Middleton $\mathrm{H}$, King N. Transfection of choanoflagellates illuminates their cell biology and the ancestry of animal septins. Mol Biol Cell. 2018. doi:10.1091/mbc.e1808-0514

5. Dayel MJ, Alegado RA, Fairclough SR, Levin TC, Nichols SA, McDonald K, et al. Cell differentiation and morphogenesis in the colony-forming choanoflagellate Salpingoeca rosetta. Dev Biol. 2011;357: 73-82. doi:10.1016/j.ydbio.2011.06.003

6. Fairclough SR, Chen Z, Kramer E, Zeng Q, Young S, Robertson HM, et al. Premetazoan genome evolution and the regulation of cell differentiation in the choanoflagellate Salpingoeca rosetta. Genome Biol. 2013;14: 1-15. doi:10.1186/gb-2013-14-2-r15

7. Levin TC, Greaney AJ, Wetzel L, King N. The Rosetteless gene controls development in the choanoflagellate S. rosetta. Elife. 2014;3. doi:10.7554/eLife.04070

8. Wetzel L, Levin T, Hulett RE, Chan D, King G, Aldayafleh R, et al. Glycosyltransferase homologs prevent promiscuous cell aggregation and promote multicellular development in the choanoflagellate \&lt;em\&gt;S. rosetta\&lt;/em\&gt; bioRxiv. 2018. Available: http://biorxiv.org/content/early/2018/08/04/384453.abstract

9. Schiwitza S, Arndt H, Nitsche F. Four new choanoflagellate species from extreme saline environments: Indication for isolation-driven speciation exemplified by highly adapted Craspedida from salt flats in the Atacama Desert (Northern Chile). Eur J Protistol. 2018;66: 86-96. doi:10.1016/j.ejop.2018.08.001

10. Richter DJ, Fozouni P, Eisen MB, King N. Gene family innovation, conservation and loss on the animal stem lineage. Elife. 2018;7. doi:10.7554/eLife.34226

11. Larson BT, Ruiz-Herrero T, Lee S, Kumar S, Mahadevan L, King N. Biophysical principles of choanoflagellate self-organization. bioRxiv. 2019; 659698. doi:10.1101/659698

12. Laundon D, Larson BT, McDonald K, King N, Burkhardt P. The architecture of cell differentiation in choanoflagellates and sponge choanocytes. PLoS Biol. 2019;17: e3000226. doi:10.1371/journal.pbio.3000226

13. Burkhardt $P$, Grønborg M, McDonald K, Sulur T, Wang Q, King N. Evolutionary insights into premetazoan functions of the neuronal protein Homer. Mol Biol Evol. 2014;31: 2342- 
2355. doi:10.1093/molbev/msu178

14. Choi HMT, Calvert CR, Husain N, Huss D, Barsi JC, Deverman BE, et al. Mapping a multiplexed zoo of mRNA expression. Development. 2016;143: 3632-3637.

doi:10.1242/dev.140137

15. DePas WH, Starwalt-Lee R, Van Sambeek L, Kumar SR, Gradinaru V, Newman DK. Exposing the three-dimensional biogeography and metabolic states of pathogens in cystic fibrosis sputum via hydrogel embedding, clearing, and rRNA labeling. MBio. 2016;7: e00796----16. doi:10.1128/mBio.00796-16

16. Pernthaler A, Pernthaler J, Amann R. Fluorescence in situ hybridization and catalyzed reporter deposition for the identification of marine bacteria. Appl Environ Microbiol. 2002;68: 3094-3101. doi:10.1128/AEM.68.6.3094-3101.2002

17. Amann RI, Binder BJ, Olson RJ, Chisholm SW, Devereux R, Stahl DA. Combination of 16S rRNA-targeted oligonucleotide probes with flow cytometry for analyzing mixed microbial populations. Appl Environ Microbiol. 1990;56: 1919-1925. Available: http://www.ncbi.nlm.nih.gov/pubmed/2200342\%0Ahttp://www.pubmedcentral.nih.gov/artic lerender.fcgi?artid=PMC184531

18. Manz W, Amann R, Ludwig W, Wagner M, Schleifer KH. Phylogenetic Oligodeoxynucleotide Probes for the Major Subclasses of Proteobacteria: Problems and Solutions. Syst Appl Microbiol. 1992;15: 593-600. doi:10.1016/S0723-2020(11)80121-9

19. Kuru E, Hughes HV, Brown PJ, Hall E, Tekkam S, Cava F, et al. In situ probing of newly synthesized peptidoglycan in live bacteria with fluorescent D-amino acids. Angew Chemie - Int Ed. 2012;51: 12519-12523. doi:10.1002/anie.201206749

20. WHIPPS JM. Effects of Mycoparasites on Sclerotia-Forming Fungi. In: BEEMSTER ABR, BOLLEN GJ, GERLAGH M, RUISSEN MA, SCHIPPERS B, TEMPEL A, editors. Biotic Interactions and Soil-Borne Diseases. Elsevier; 1991. pp. 129-140. doi:https://doi.org/10.1016/B978-0-444-88728-3.50025-1

21. Shapira M. Gut Microbiotas and Host Evolution: Scaling Up Symbiosis. Trends Ecol Evol. 2016;31: 539-549. doi:10.1016/j.tree.2016.03.006

22. Roeselers G, Mittge EK, Stephens WZ, Parichy DM, Cavanaugh CM, Guillemin K, et al. Evidence for a core gut microbiota in the zebrafish. ISME J. 2011;5: 1595-1608. doi:10.1038/ismej.2011.38

23. Huse SM, Ye Y, Zhou Y, Fodor AA. A core human microbiome as viewed through $16 \mathrm{~S}$ rRNA sequence clusters. PLoS One. 2012;7. doi:10.1371/journal.pone.0034242

24. Doolittle WF, Booth A. It's the song, not the singer: an exploration of holobiosis and evolutionary theory. Biol Philos. 2017;32: 5-24. doi:10.1007/s10539-016-9542-2

25. Alegado RA, Brown LW, Cao S, Dermenjian RK, Zuzow R, Fairclough SR, et al. A bacterial sulfonolipid triggers multicellular development in the closest living relatives of animals. Elife. 2012;2012. doi:10.7554/eLife.00013 
26. Woznica A, Gerdt JP, Hulett RE, Clardy J, King N. Mating in the Closest Living Relatives of Animals Is Induced by a Bacterial Chondroitinase. Cell. 2017;170: 1175-1183.e11. doi:10.1016/j.cell.2017.08.005

27. Woznica A, Cantley AM, Beemelmanns C, Freinkman E, Clardy J, King N. Bacterial lipids activate, synergize, and inhibit a developmental switch in choanoflagellates. Proc Natl Acad Sci U S A. 2016;113: 7894-7899. doi:10.1073/pnas.1605015113

28. Ireland E V, Woznica A, King N. Synergistic cues from diverse bacteria enhance multicellular development in a choanoflagellate. Appl Environ Microbiol. 2020;86.

29. Bayer T, Neave MJ, Alsheikh-Hussain A, Aranda M, Yum LK, Mincer T, et al. The microbiome of the red sea coral Stylophora pistillata is dominated by tissue-associated Endozoicomonas bacteria. Appl Environ Microbiol. 2013;79: 4759-4762. doi:10.1128/aem.00695-13

30. Beinart RA, Nyholm S V, Dubilier N, Girguis PR. Intracellular Oceanospirillales inhabit the gills of the hydrothermal vent snail Alviniconcha with chemosynthetic, \$Y \$-Proteobacterial symbionts. Environ Microbiol Rep. 2014;6: 656-664. doi:10.1111/1758-2229.12183

31. Carlos C, Torres TT, Ottoboni LMM. Bacterial communities and species-specific associations with the mucus of Brazilian coral species. Sci Rep. 2013;3. doi:10.1038/srep01624

32. Dishaw LJ, Flores-Torres J, Lax S, Gemayel K, Leigh B, Melillo D, et al. The gut of geographically disparate Ciona intestinalis harbors a core microbiota. PLoS One. 2014;9: e93386. doi:10.1371/journal.pone.0093386

33. Goffredi SK, Orphan VJ, Rouse GW, Jahnke L, Embaye T, Turk K, et al. Evolutionary innovation: A bone-eating marine symbiosis. Environ Microbiol. 2005;7: 1369-1378. doi:10.1111/j.1462-2920.2005.00824.x

34. Kurahashi M, Yokota A. Endozoicomonas elysicola gen. nov., sp. nov., a \$y \$proteobacterium isolated from the sea slug Elysia ornata. Syst Appl Microbiol. 2007;30: 202-206. doi:10.1016/j.syapm.2006.07.003

35. Morrow KM, Moss AG, Chadwick NE, Liles MR. Bacterial Associates of Two Caribbean Coral Species Reveal Species-Specific Distribution and Geographic Variability. Appl Environ Microbiol. 2012;78: 6438-6449. doi:10.1128/aem.01162-12

36. Vezzulli L, Pezzati E, Huete-Stauffer C, Pruzzo C, Cerrano C. 16SrDNA Pyrosequencing of the Mediterranean Gorgonian Paramuricea clavata Reveals a Link among Alterations in Bacterial Holobiont Members, Anthropogenic Influence and Disease Outbreaks. PLoS One. 2013;8: e67745. doi:10.1371/journal.pone.0067745

37. Bayer C, Heindl NR, Rinke C, Lücker S, Ott JA, Bulgheresi S. Molecular characterization of the symbionts associated with marine nematodes of the genus Robbea. Environ Microbiol Rep. 2009;1: 136-144. doi:10.1111/j.1758-2229.2009.00019.x

38. Payne $\mathrm{SH}$, Loomis WF. Retention and loss of amino acid biosynthetic pathways based on analysis of whole-genome sequences. Eukaryot Cell. 2006;5: 272-276. 
doi:10.1128/EC.5.2.272-276.2006

39. Rinke C, Schmitz-Esser S, Stoecker K, Nussbaumer AD, Molnar DA, Vanura K, et al. "Candidatus Thiobios zoothamnicoli", an ectosymbiotic bacterium covering the giant marine ciliate Zoothamnium niveum. Appl Environ Microbiol. 2006;72: 2014-2021. doi:10.1128/AEM.72.3.2014-2021.2006

40. Tian RM, Wang Y, Bougouffa S, Gao ZM, Cai L, Bajic V, et al. Genomic analysis reveals versatile heterotrophic capacity of a potentially symbiotic sulfur-oxidizing bacterium in sponge. Environ Microbiol. 2014;16: 3548-3561. doi:10.1111/1462-2920.12586

41. Woznica A, Kumar A, Sturge CR, Xing C, King N, Pfeiffer K. STING mediates immune responses in a unicellular choanoflagellate. bioRxiv. 2021; 6.

doi:10.1101/2021.05.13.443778

42. Hug LA, Baker BJ, Anantharaman K, Brown CT, Probst AJ, Castelle CJ, et al. A new view of the tree of life. Nat Microbiol. 2016;1: nmicrobiol201648.

doi:10.1038/nmicrobiol.2016.48 